585. Eug. Lellmann und Rud. Grothmann: Ueber einige Derivate der Salicylsäure.

(Eingegangen am 21. Norember; mitgetheilt in der Sitzung von Hrn. A. Pinner.)

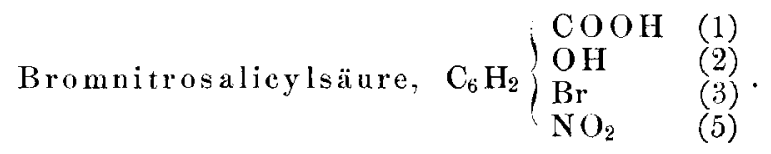

Die Substitution von Brom für die Amidogruppe in der von Babcock ${ }^{1}$ ) dargestellten Nitroamidosalicylsäure ist eine wenig glattverlaufende Reaktion. Die Diazoverbindung liefert bei der Behandlung mit Bromwasserstoffsäure sehr schlechte Resultate, nicht viel besser kommt man fort, wenn ein Gemisch von Bromwasserstoffsäure und Eisessig verwendet wird; etwas bessere Ausbeute liefert folgendes Verfahren: Man übergiesst die Nitroamidosalicylsäure in kleinen Portionen mit dem Vierfachen der berechneten Menge Bromwasserstoffsäure, löst das entstandene Bromhydrat in viel Eisessig und lässt kurze Zeit die Blasen von $\mathrm{N}_{2} \mathrm{O}_{3}$ die Flüssigkeit passiren, sodann erwärmt man, bis kein Stickstoff mehr entweicht, verdünnt mit Wasser und bringt vorsichtig zur Trockne; der Rückstand wird mit wenig warmem Wasser behandelt, wobei die Bromnitrosalicylsäure in Lösung geht, während ein unten zu beschreibendes Dibromnitrophenol zurückbleibt. Durch wiederholtes Umkrystallisiren aus Wasser gelingt die Reinigung der Säure ohne Schwierigkeit.

Her $z \circ g^{2}$ ) hat dieselbe Säure durch Bromirung von Nitrosalicylsäure (Schmp. 228 ) im Rohre bei 130-140 dargestellt; bei Weitem zweckmässiger ist folgendes Verfahren: Man löst die Nitrosalicylsäure in der erforderlichen Menge heissen Eisessigs und setzt etwas mehr als die berechnete Quantität Brom hinzu; nach dem Erkalten vertreibt man das überschüssige Brom auf dem Wasserbade, versetzt mit Wasser und destillirt im Dampfstrome, um etwa gebildete Phenole zu entfernen. Hierauf wird das im Destillirgefässe zurückbleibende Oel öfters mit Wasser ausgekocht, wodurch die Bromnitrosalicylsäure in Lösung geht; der unlösliche Rückstand besteht aus derselben Verbindung, die bei der Darstellung der Säure auf dem Diazowege erhalten wird; die Ausbeute an Bromnitrosalicylsäure ist befriedigend. Die auf die eine oder andere Weise bereitete Säure ist leicht löslich in heissem, schwerer in kaltem Wasser, krystallisirt aus verdünnten Lösungen in farblosen Nädelchen, aus concentrirten in

1) Diese Berichte XII, 1345; Dissertation Göttingen 1879.

2) Dissertation Göttingen 1879. 
Warzen; sie löst sich sehr leicht in Alkohol und Aether und schmilzt bei $222^{0}$.

$\begin{array}{ccc} & \text { Gefunden } & \text { Berechnet } \\ \mathrm{C} & 31.82 & 32.07 \mathrm{pCt} . \\ \mathrm{H} & 1.90 & 1.53 \% \\ \mathrm{~N} & 5.21 & 5.34 \%\end{array}$

Halbbaryumbromnitrosalicylat, $\left(\mathrm{C}_{6} \mathrm{H}_{2} \mathrm{Br} \mathrm{N} \mathrm{O} \mathrm{O}_{2} \mathrm{OHCOO}\right)_{2} \mathrm{Ba}+4 \mathrm{H}_{2} \mathrm{O}$

wird erhalten aus Baryumcarbonat und der Säure und bildet lange gelbe Nadeln.
$\mathrm{H}_{2} \mathrm{O}$
Gefunden
Berechnet
9.69
$9.85 \mathrm{pCt}$.

Die Baryumbestimmung des wasserfreien Salzes ergab:

$$
\begin{array}{ccc} 
& \text { Gefunden } & \text { Berechnet } \\
\text { Ba } & 20.72 & 20.79 \text { pCt. }
\end{array}
$$

Halbcalciumbromnitrosalicylat, $\left(\mathrm{C}_{6} \mathrm{H}_{2} \mathrm{Br} \mathrm{N} \mathrm{O} \mathrm{OH} \mathrm{OOO}_{2} \mathrm{Ca}+6 \mathrm{H}_{2} \mathrm{O}\right.$

krystallisirt in gelben Säulen.

$$
\begin{aligned}
& \text { Gefunden } \\
& \mathrm{H}_{2} \mathrm{O} \quad 16.00 \\
& \text { Gefunden im } \\
& \text { entwässerten Salze } \\
& \mathrm{Ca} \quad 7.03 \\
& \text { Berechnet } \\
& 16.12 \text { pCt. } \\
& \text { Berechnet } \\
& 7.12 \text { pCt. } \\
& \text { Bromamidosalicylsäure, } \mathrm{C}_{6} \mathrm{H}_{2} \mid \begin{array}{ll}
\mathrm{COOH} & (1) \\
\mathrm{OH} & (2) \\
\mathrm{Br} & (3) \\
\mathrm{NH}_{2} & (5)
\end{array} \text {. }
\end{aligned}
$$

Die eben beschriebene Säure liefert bei der Reduktion mit Zinn und Salzsäure eine Bromamidosalicylsäure, deren Chlorhydrat man beim Eindampfen der entzinnten Lösung in farblosen Nadeln erhält. Aus diesem Salze lässt sich die Säure nicht frei machen, da sie schnell zu unansehnlichen Massen verharzt. Die salzsaure Amidosäure löst sich leicht in Wasser und Alkohol, fast gar nicht in Aether.

$\begin{array}{ccc} & \text { Gefunden } & \text { Berechnet } \\ \mathrm{C} & 30.93 & 31.25 \mathrm{pCt} \\ \mathrm{H} & 3.62 & 2.61 \% \\ \mathrm{~N} & 5.38 & 5.22 \%\end{array}$

Die Darstellung eines Platinchloriddoppelsalzes gelang nicht.

$$
\text { Bromsalicylsäure, } \mathrm{C}_{6} \mathrm{H}_{3} \begin{cases}\mathrm{COOH} & \text { (1) } \\ \mathrm{OH} & \text { (2). } \\ \mathrm{Br} & \text { (3) }\end{cases}
$$

Die salzsaure Bromamidosalicylsäure liefert in concentrirter wässriger Lösung mit Natriumnitrit einen Diazokörper, der sich in 
gelbbraunen glänzenden Blättchen abscheidet; man filtrirt sotort ab und trocknet $z$ wischen Fliesspapier. Die Diazoverbindung verpufft beim Erhitzen und nimmt beim Liegen an der Luft eine dunkelbraune Farbe an; die Substanz wurde nicht näher untersucht, sondern gleich durch anhaltendes Kochen mit absolutem Alkohol zerlegt. Die Umsetzung ist beendet, sobald der Diazokörper vollständig in Lösung gegangen ist, man destillirt sodann den Alkohol ab, fügt, Wasser zum Rückstande und leitet, um einige Beimengungen, die in Bromphenolen zu bestehen scheinen, zu entfernen, Wasserdampf ein. Da aus dem Diazoamidokörper neben zu erwartender Bromsalicylsäure auch Bromamidosalicylsäure entstehen musste, so konnte es nicht befremden, dass eine harzige Masse resultirte, der jedoch durch wiederholtes Auskochen mit Wasser nicht unbeträchtliche Mengen Bromsalicylsäure entzogen werden konnten. Diese Säure liess sich durch Zerlegung des Bleisalzes mit Schwefelwasserstoff leicht in ganz reinem Zustande erhalten; sie bildet Nadeln, die in kaltem Wasser ziemlich schwer, leichter in heissem, sehr leicht in Alkohol löslich sind. Der Schmelzpunkt wurde bei $184^{\circ}$ beobaehtet.

$\begin{array}{lcc} & \text { Gefunden } & \text { Berechnet } \\ \text { C } & 38.85 & 38.71 \text { pCt. } \\ \text { H } & 3.06 & 2.30 \% \\ \mathrm{Br} & 36.89 & 36.87 \%\end{array}$

Hübner und Heinzerling ${ }^{1}$ ) erhielten aus der Bromamidobenzö̈säure $\mathrm{C}_{6} \mathrm{H}_{3} \mathrm{CO} \stackrel{\text { I. } O H ~ N \mathrm{H}_{2}}{\mathrm{III}} \mathrm{Br}$ durch Diazotiren eine Bromsalicylsäure, die mit der von uns dargestellten identisch sein müsste, eigenthümlicher Weise differiren die Schmelzpunkte bedentend; Hübner und Heinzerling fanden den Schmelzpunkt $219-220^{\circ}$ und erhielten ein Bleisalz, durch Fällıng des Ammonsalzes mit Bleiacetat, von der Formel $\left(\mathrm{C}_{6} \mathrm{H}_{3} \mathrm{BrOHCOO}\right)_{2} \mathrm{~Pb}$, während das Baryumsalz der von uns dargestellten Säure mit Bleiacetat ein Salz

$$
\mathrm{C}_{6} \mathrm{H}_{3} \mathrm{Br} \underset{\mathrm{O}}{\mathrm{COO}}=\mathrm{Pb}
$$

lieferte.

Um die Constitution unserer Bromsalicylsäure festzustellen, haben wir dieselbe mit Wasser $5-6$ Stunden lang auf $180^{\circ}$ erhitzt; es tritt Kohlensäure aus und man erhält $o$-Bromphenol, welches durch Destillation mit Wasserdampf leicht gereinigt werden kann. Da die Menge zu einer Siedepunktbestimmung nicht ausreichte, so wurde das Bromphenol, in Eisessig gelöst, mit einem Gemisch von gleichen Theilen rauchender Salpetersäure und Eisessig unter Vermeidung einer Temperaturerhöhung nitrirt, und das resultirende Bromdinitrophenol

1) Zeitschrift für Chemie 1871, 709. 
durch seinen Schmelzpankt $119^{\circ}$ mit dem von Körner ${ }^{1}$ ) erhaltenen identificirt.

$$
\begin{array}{ccc} 
& \text { Gefuuden } & \text { Berechnet } \\
\mathrm{N} & 10.92 & 1065 \mathrm{pCt} .
\end{array}
$$

Halbbaryumbromsalicylat, $\left(\mathrm{C}_{6} \mathrm{H}_{3} \mathrm{BrOHCOO}\right)_{2} \mathrm{Ba}+3 \mathrm{H}_{2} \mathrm{O}$, aus Baryumcarbonat und der Säure dargestellt, bildet schwach röthlich gefärbte Prismen.

$\begin{array}{ccc} & \text { Gefunden } & \text { Berechnet } \\ \mathrm{H}_{2} \mathrm{O} \quad 8.69 & 8.67 \mathrm{pCt} . \\ \text { Gefunden } & \text { Berechnet } \\ \text { im entwässerten Salze } & \\ \mathrm{Ba} \quad 23.64 & 24.08 \mathrm{pCt} .\end{array}$

Halbcalciumbromsalycilat, $\left(\mathrm{C}_{6} \mathrm{H}_{3} \mathrm{BrOHCOO}\right)_{2} \mathrm{Ca}+12 \mathrm{H}_{2} \mathrm{O}$, erhältlich aus der Säure und Calciumcarbonat; das Salz ist sehr leicht löslich in Wasser und krystallisirt in Warzen, deren Krystallwassergehalt jedoch wegen der leichten Zersetzlichkeit nicht mit Sicherheit festgestellt werden konnte.

Das lufttrockene Salz ergab:

$$
\begin{aligned}
& \text { Berechnet } \\
& \begin{array}{lll}
\mathrm{Ca} & 5.65 & 5.81 \mathrm{pCt} \text {. }
\end{array} \\
& \text { Bleibromsalicylat, } \mathrm{C}_{6} \mathrm{H}_{3} \mathrm{Br}_{-} \mathrm{COO} \cdot \mathrm{Pb} \text {. }
\end{aligned}
$$

Versetzt man die wässrige Lösung des Baryumsalzes mit Bleiacetat, so fällt neutrales Bleibromsalicylat als schwach gelblich gefärbter Niederschlag aus.

$$
\begin{array}{cc}
\multicolumn{1}{c}{\begin{array}{c}
\text { Berechnet } \\
\mathrm{Pb} 49.16
\end{array}} & \begin{array}{c}
\text { Gefunden } \\
49.05 \mathrm{pCt} .
\end{array} \\
\text { Dibromsalicylsä ure, } \mathrm{C}_{6} \mathrm{H}_{2}\left\{\begin{array}{ll}
\mathrm{COOH} & (1) \\
\mathrm{OH} & (2) \\
\mathrm{Br} & (3) \\
\mathrm{Br} & (5)
\end{array} .\right.
\end{array}
$$

Der oben erwähnte Diazokörper wurde mit wenig concentrirter Bromwasserstoffsäure so lange gelinde erwärmt, bis kein Stickstoff mehr entwich, und das Produkt zur Reinigung mit Wasserdämpfen behandelt; hierbei gingen nicht unbeträchtliche Mengen von Substanzen über, aus denen nichts Charakterisirtes gewonnen werden konnte.

1) Jahresbericht für Chem. 1875, 335. 
Man filtrirte sodann heiss von einem theerigen Rückstande $a b$ und erhielt beim Erkalten des Filtrats eine Abscheidung einer geringen Menge sehr unreiner Dibromsalicylsäure. Zum Zwecke der Reinigung wurde das Baryumsalz dargestellt, die Lösung desselben eingetrocknet und dem Rückstande das Salz mit Alkohol entzogen; man dampfte hierauf wieder zur Trockne, löste den Rückstand in Wasser und schlug mit Bleiacetat das Bleisalz nieder, letzteres wurde zur Reinigung mit Alkohol ausgekocht und dann mit Schwefelwasserstoff zerlegt; auf diesem umständlichen Wege gelangte man zu einer reinen Süure rom Schmelzpunikte $223^{\circ}$.

Viel leichter lässt sich dieselbe Säure durch Bromiren von Salicylsäure gewinnen; zu diesem Zwecke lässt man zu einer dünnen Lösung von Salicylsäure in Eisessig, die durch kaltes Wasser gekühlt wird, nach und nach ein Viertel mehr als die zur Bildung von Dibromsalicylsäure erforderliche Menge Brom binzutropfen; vortheilhaft ist es, auch das Brom mit einem gleichen Volumen Eisessig zu verdünnen. Nachdem das Gemisch einige Stunden sich selbst überlassen ist, fällt man mit Wasser und wäscht das abgeschiedene Produkt, dessen Schmelzpunkt zwischen $190-200^{\circ}$ liegt, nach. Zur Entfernung von etwa vorhandenen flüchtigen Substanzen wurde längere Zeit mit Wasser gekocht, die Flüssigkeit hierauf rom Rückstande abgegossen und die zurückgebliebene Säure in das Barytsalz übergeführt; aus letzterem regenerirte man die Säure als eine in langen, farblosen Nadeln krystallisirende, bei $223^{\circ}$ schmelzende Substanz, die sich schwer in kochendem, fast gar nicht in kaltem Wasser löst.

$$
\begin{array}{ccc} 
& \text { Gefunden } & \text { Berechnet } \\
\mathrm{Br} & 54.38 & 54.05 \text { pCt. }
\end{array}
$$

Halbbaryumdibromsalicylat, $\left(\mathrm{C}_{6} \mathrm{H}_{2} \mathrm{Br}_{2} \mathrm{OHCOO}\right)_{2} \mathrm{Ba}+4 \mathrm{H}_{2} \mathrm{O}$, krystallisirt in langen, farblosen Nadeln.

$$
\begin{array}{ccr} 
& \text { Gefunden } & \text { Berechnet } \\
\mathrm{H}_{2} \mathrm{O} \quad 9.28 & 9.01 \mathrm{pCt} . \\
\multicolumn{2}{c}{\text { Gefunden }} & \text { Berechnet } \\
\mathrm{im} \text { entwässerten Salze } & 18.85 \mathrm{pCt} .
\end{array}
$$

$$
\text { Bleidibromsalicylat, } \mathrm{C}_{6} \mathrm{H}_{2} \mathrm{Br}_{2}-\mathrm{COO}_{0}, \mathrm{~Pb}
$$

wird als weisser, in Wasser unlöslicher Niederschlag beim Fällen des Baryumsalzes mit Bleiacetat erhalten.
Gefunden
Berechnet
$\mathrm{Pb} \quad 42.27$
$41.31 \mathrm{pCt}$. 


$$
\text { Bromnitrosalicylsäıre, } \mathrm{C}_{6} \mathrm{H}_{2}\left\{\begin{array}{ll}
\mathrm{COOH} & (1) \\
\mathrm{OH}^{2} & (2) \\
\mathrm{NO}_{2} & (3) \\
\mathrm{Br}^{2} & (5)
\end{array} .\right.
$$

Monobromsalicylsäure rom Schmp. $165^{\circ}$, die wir nach Hübner und Heinzerling ${ }^{1}$ ) durch Bromirung von Salicylsäure dargestellt hatten, wurde in folgender Weise in Bromnitrosalicylsäure übergeführt: man löste $5 \mathrm{~g}$ Bromsalicylsäure in $30 \mathrm{~g}$ Eisessig und goss diese Lösung in $25 \mathrm{~g}$ rauchender Salpetersäure, die mit einem gleichen Volumen Eisessig verdünnt und auf $0^{0}$ abgekühlt war; das Säuregemisch blieb dann zwei Stunden stehen und wurde hierauf mit dem 5 fachen Volumen kalten Wassers versetzt, es schieden sich sogleich hellgelbe Flocken aus, die abfiltrirt und mit wenig Wasser gewaschen wurden. Das Produkt behandelten wir längere Zeit mit Wasserdampf, um etwa entstandene Phenolderivate zu entfernen; im Kühlrohr sammelte sich in der That eine kleine Menge einer gelben Substanz an, aus welcher jedoch keine charakterisirte Verbindung gewonnen werden konnte. Aus dem Destillationsrückstande schied sich beim Erkalten eine ansebnliche Quantität der Bromnitrosalicylsäure in gelben Nadeln aus, die bei $174^{\circ}$ schmolzen und fast ganz rein waren, denn nach wiederholtem Umkrystallisiren stieg der Schmelzpunkt nur auf $175^{\circ}$. Die neue Säure ist leicht löslich in heissem, schwerer in kaltem Wasser und krystallisirt daraus in gelblichen, zu Gruppen vereinigten, kurzen Nadeln, die ein Molekül Krystallwasser enthalten; letzteres entweicht nicht beim Liegen an der Luft, wohl aber langsam über Schwefelsäure, schnell bei $100-110^{\circ}$. Die Säure bildet vorwiegend neutrale Salze, die meist anhydritisch sind und durch verdünnte Essigsäure in die sauren übergeführt werden können.

\section{Halbbaryumbromnitrosalieylat, $\left(\mathrm{C}_{6} \mathrm{H}_{2} \mathrm{BrNO}_{2} \mathrm{OHCOO}\right)_{2} \mathrm{Ba}$.}

Dieses Salz entsteht, wenn man die wässrige Lösung der Säure mit Barytwasser versetzt; letzteres fügt man so lange hinzu, als der anfangs entstehende dunkelrothe Niederschlag von neutralem Salze noch gelöst wird; einen etwa hinzugegebenen Ueberschuss kann man durch Erhitzen mit etwas Essigsäure fortnehmen. Das Salz krystallisirt in röthlich gelben, verwachsenen Nadeln.

$$
\begin{array}{ccc} 
& \text { Gefunden } & \text { Berechnet } \\
\mathrm{Ba} & 20.62 & 20.79 \mathrm{pCt} .
\end{array}
$$

Halbealciumbromitrosalicylat,

$$
\left(\mathrm{C}_{6} \mathrm{H}_{2} \mathrm{BrN} \mathrm{O}_{2} \mathrm{OHCOO}\right)_{2} \mathrm{Ca}+\mathrm{xH}_{2} \mathrm{O} \text {, }
$$

wurde erhalten aus der Säure und Calciumcarbonat; das Filtrat erhitzt man auch in diesem Falle mit etwas Essigsäure. Das Salz ist sehr 
leicht löslich in Wasser und scheidet sich flockig aus seiner Lösung ab. Der Krystallwassergehalt scheint ein hoher zu sein, lässt sich jedoch wegen der aufserordentlich grossen Verwitterbarkeit des Salzes nicht bestimmen.

Im entwässerten Salze fanden sich:

$$
\begin{array}{ccc} 
& \text { Gefunden } & \text { Berechnet } \\
\mathrm{Ca} & 7.09 & 7.12 \mathrm{pCt} .
\end{array}
$$

Halbbleibromnitrosalicylat, $\left(\mathrm{C}_{6} \mathrm{H}_{2} \mathrm{BrNO}_{2} \mathrm{OHCOO}\right)_{2} \mathrm{~Pb}$.

Versetzt man eine wässrige Lösung der Säure mit Bleiacetatlösung, so fällt neutrales Bleisalz als gelber Niederschlag aus; durch Auflösen desselben in warmer verdünnter Essigsäure erhält man beim Erkalten das saure Salz in gelben, unter sich verwachsenen Nadeln.

$$
\begin{array}{ccc} 
& \text { Gefunden } & \text { Berechnet } \\
\mathrm{Pb} & 28.13 & 28.39 \\
\text { pCt. }
\end{array}
$$

Baryumbromnitrosalicylat, $\mathrm{C}_{6} \mathrm{H}_{2} \mathrm{BrNO}_{2}<\mathrm{O}_{-}^{\mathrm{COO}}>\mathrm{Ba}+2 \mathrm{H}_{2} \mathrm{O}$, bildet sich in der oben angegebenen Weise; man filtrirt ab, löst in heissem Wasser und erhält nach dem Concentriren der Lösung das Salz in purpurrothen, harten Krusten.

$$
\text { Gefunden }
$$
$\mathrm{H}_{2} \mathrm{O}$
$9.17 \quad 8.47$
$8.31 \mathrm{pCt}$.

Berechnet

Im entwässerten Salze:

$$
\begin{array}{ccc} 
& \text { Gefunden } & \text { Berechnet } \\
\text { Ba } & 34.54 & 34.51 \text { pCt. }
\end{array}
$$

Bleibromnitrosalicylat, $\mathrm{C}_{6} \mathrm{H}_{2} \mathrm{BrNO}_{2} \underset{\mathrm{O}-\ldots \mathrm{Pb},}{\mathrm{COO}}$ in Wasser kaum löslicher Niederschlag.

$$
\begin{array}{ccc} 
& \text { Gefunden } & \text { Berechnet } \\
\mathrm{Pb} & 44.23 & 44.32 \mathrm{pCt} .
\end{array}
$$

Um die Constitution dieser Säure festzustellen, verfuhr man folgendermaassen: einige Gramme der Verbindung wurden mit Wasser 5-6 Stunden lang im Rohr auf $210^{\circ}$ erhitzt und das Produkt sodann mit Wasserdampf destillirt, im Kühlrohr setzte sich bald eine schwefelgelbe krystallinische Verbindung ab, die nach einmaligem Umkrystalliin Ir. Iv. siren aus verdünntem Alkohol als das Bromuitrophenol $\mathrm{C}_{6} \mathrm{H}_{3} \mathrm{OHNO}_{2} \mathrm{Br}$ vom Schmelzpunkte $88^{\circ}$ erkannt wurde. Demnach kommt der Bromnitrosalicylsäure die angegebene Constitution zu. 


$$
\text { Dibromnitrophenol, } \mathrm{C}_{6} \mathrm{H}_{2}\left\{\begin{array}{ll}
\mathrm{OH} & (1) \\
\mathrm{Br} & (2) \\
\mathrm{NO}_{2} & (4) \\
\mathrm{Br} & (6)
\end{array}\right. \text {. }
$$

Das oben erwähnte, bei beiden Bereitungsweisen der Bromnitrosalicylsäure auftretende Nebenprodukt ist ein Dibromnitrophenol, dem man unter der Annahme, dass die Carboxylgruppe durch Brom verdrängt wurde, die angegebene Formel zuschreiben darf. Ist die Verbindung dureh Umkrystallisiren aus Alkohol gereinigt, so schmilzt sie bei $142^{0}$, sie ist schwer löslich in Wasser, leichter in Alkohol, aus dem sie in feinen Nadeln anschiesst.

$\begin{array}{ccc} & \text { Gefunden } & \text { Berechnet } \\ \mathrm{C} & 24.19 & 24.24 \text { pCt. } \\ \mathrm{H} & 1.40 & 1.01 \text { » }\end{array}$

Baryumdibromnitrophenolat, $\left(\mathrm{C}_{6} \mathrm{H}_{2} \mathrm{Br}_{2} \mathrm{NO}_{2} \mathrm{O}\right)_{2} \mathrm{Ba}+10 \mathrm{H}_{2} \mathrm{O}$, erhalten aus dem Phenol und Baryumearbonat, bildet grüne Nadeln. Concentrirte Lösungen des Salzes zeigen eine braune, verdünnte eine grüne Farbe.

$\begin{array}{ccc} & \text { Gefunden } & \text { Berechnet } \\ \mathrm{H}_{2} \mathrm{O} & 19.51 & 19.80 \mathrm{pCt} .\end{array}$

Im entwässerten Salze, welches braun erscheint, fanden sich $18.58 \mathrm{pCt}$. Ba, während die Theorie $18.79 \mathrm{pCt}$. verlangt.

Dibromamidophenol, $\mathrm{C}_{6} \mathrm{H}_{2} \mathrm{OHBr} 2 \mathrm{NH}_{2}$.

Das Dibromnitrophenol lässt sich mit Zinn und Salzsäure nur schwierig amidiren, daher verwendet man bequemer Zinn und Eisessig als Agentien. Nach der Entfernung des Zinns durch Schwefelwasserstoff krystallisirt das Dibromamidophenol in schwach röthlich gefärbten Nadeln aus, die gereinigt bei $190^{\circ}$ sehmelzen und sich, über den Schmelzpunkt hinaus erhitzt, zersetzen. Die Verbindung ist schwer Iöslieh in kaltem Wasser, leichter in heissem, noch leichter in Alkohol und liefert keine Salze.

$\begin{array}{ccc} & \text { Gefundel } & \text { Berechnet } \\ \mathrm{N} & 5.25 & 5.24 \text { pCt. } \\ \mathrm{Br} & 59.78 & 59.93 \text { \% }\end{array}$

Die Constitution ergiebt sich aus dem Gesagten.

Die im Vorstehenden beschriebenen Versuche wurden vor ca. vier Jahren im Universitätslaboratorium zu Göttingen ausgeführt. 